\title{
An Exact Solution for the Infinite Horizon LQ Optimal Output Tracking Problem
}

\author{
Peter Bauer*, Balazs Kulcsar** and Jozsef Bokor*
}

\begin{abstract}
The paper proposes a new, discrete time LQ optimal controller synthesis for output tracking over an infinite horizon. The resulted controller consists of a state feedback and a feedforward term. The solution requires a one step ahead prediction of the reference signal. For a smooth enough reference signal this can be replaced with extrapolation. Here, the properties for constant reference signals are examined, time varying references will be covered with another article. The method guarantees asymptotical stability and zero steady state tracking error. The elaborated techniques satisfy the separation principle for an arbitrary deterministic or stochastic state estimator.

The reference tracking problem for a quadrotor helicopter is solved by the method. The trajectory tracking control is successfully applied both with reference signal preview and extrapolation (considering time varying references also).
\end{abstract}

Index Terms - output tracking, infinite horizon, discrete time LQ, constant reference signal

\section{INTRODUCTION}

LQ optimal state and output tracking control have been an actively researched field since 1960s. Several exact or approximate solutions were derived using different approaches. The existing methods can be classified in two main groups, such as a priori given reference signals over the known future horizon and solutions using only instantaneous and past reference values.

The first group is given as the predictive and preview techniques. Most of the predictive techniques are summarized in [6], [7] and [9] uses LQ optimal approaches. These methodologies are out of interest if the reference signal is unknown.

On the other hand, output tracking problems can be solved using past and current measurement and state information. This solution does not consider the future evaluation of the trajectory.

If the states associated to the reference signal is covered in the kernel of the $A$ system matrix, the technique is called tracking via coordinate translation [5]. Usually, this condition can not be fulfilled.

The authors gratefully acknowledge the support of the Hungarian National Science foundation (OTKA N: K60767) and the Hungarian National Office for Research and Technology through the project "Advanced Vehicles and Vehicle Control Knowledge Center" (NKTH RET04/2004).

*Faculty of Transportation Engineering, Department of Control and Transport Automation, Budapest University of Technology and Economics (BUTE), Budapest, H-1111, Hungary bauer.peter@mail.bme. hu,bokor@sztaki. hu

**Delft Center for System and Control, Faculty 3ME, Technical University of Delft,Mekelweg 2, 2628CD, Delft, Netherland b.a.kulcsaretudelft.nl
Moreover, feedforward control in [5] is very sensitive to model mismatches and can only exactly determined if the number of the outputs equals the number of inputs.

LQ Servo (integral) control in [5] requires the implementation of additional integrators. These new states are related to the output error terms.

An alternate technique in [1] deals with continuous time (CT) output tracking, considering linear time varying (LTV) finite horizon and linear time invariant (LTI) large horizon solutions. The finite horizon solution is rigorously derived and contains state feedback with an extra forcing function. Both the LTV and the LTI optimal solutions have to be calculated backward in time, so these approaches need the reference signal in advance. Nevertheless, the large horizon solution is only an approximation, it does not consider infinite time. The solution assumes to have a constant reference signal. A discrete time representation is derived for the same problems in [2].

The large horizon technique proposed in [1] and [2] is further improved in [10] and [11].

In [10], the CT infinite horizon problem for a constant reference tracking has been elaborated but asymptotical tracking can not be guaranteed with.

In [11], the authors derive a system of algebraic equations based on the initial state of the forcing function (see also [1]). The backward recursion is avoided, but the structure of the reference signal has to be fixed (assumed to be polynomial).

In [8] (section 5.8-2) a 2-DOF LQ tracking solution is derived very similar to the one being presented here, but it requires a square plant and zero initial states. These limitations are not required in our solution. Another difference is the use of input (here) instead of input differences which means the avoidance of integral action in the loop. This simplifies the resulting control law.

So, the paper suggests an exact and infinite horizon output tracking solution for DT, LTI systems. The most important advantage of it is to guarantee asymptotic stability and zero steady state tracking error for constant reference output signal. The solution can be given in closed form assuring the easy implementation. The controller reflects the usual two degree of freedom structure. If needed, a state estimator can be added to it, with which it fulfills the separation principle. Properties for time varying references will be examined in a future article.

The paper is organized as follows. In section II the problem is formulated. In section III, a DT, finite horizon output tracking solution for LTI systems is derived, which is the basis for the derivation of the infinite horizon case. In section 
IV, the DT, infinite horizon solution is derived from the finite horizon case, using an additional condition (zero steady state tracking error). In section $\mathrm{V}$, the properties of the derived infinite horizon solution for constant reference signals are stated and proved. Section VI shows a potential application domain, considering the trajectory tracking control of a quadrotor helicopter (demonstrating applicability for time varying references also). Finally, section VII concludes the paper.

\section{PRELIMINARIES}

The state dynamic and measurement equations for the examined class of DT, LTI systems are given by:

$$
\begin{aligned}
x_{k+1} & =A x_{k}+B u_{k} \\
y_{k} & =C x_{k}
\end{aligned}
$$

where $x_{k} \in \mathbb{R}^{n}, u_{k} \in \mathbb{R}^{m}, y_{k} \in \mathbb{R}^{p}, A \in \mathbb{R}^{n \times n}, B \in$ $\mathbb{R}^{n \times m}, C \in \mathbb{R}^{p \times n}$.

Assume that, the pair $(A, B)$ is stabilizable and the pair $(C, A)$ is detectable.

The solution of the tracking problem depends on the information that one can gain from the measurement equation (1). Hence, not every single outputs are tracked. Moreover, the reference tracking problem can be formulated for a not directly measured artificial output.

This is the reason to introduce a new output vector $y_{k}^{r}$ strictly containing which has to be followed. $y_{k}^{r}$ can be defined separately from $y_{k}$ using another output matrix:

$$
y_{k}^{r}=C_{r} x_{k}
$$

Let the dimension of this output be $r \leq m$ and so $C_{r} \in \mathbb{R}^{r \times n}$ to assure the exact solution of the tracking problem.

\section{THE DT, FINITE HORIZON, LQ OPTIMAL OUTPUT TRACKING SOLUTION}

The goal of this section is to derive a DT finite horizon LQ optimal output tracking solution for LTI systems considering (1) and (2). The outcome of the finite horizon LTI solution will then be used for the derivation of infinite horizon results. Consider an output reference signal $r_{k} \in \mathbb{R}^{r}$ and define the tracking error as $e_{k}=y_{k}^{r}-r_{k}$. The cost function to be minimized is written as:

$$
\begin{aligned}
& J(\bar{y}, e, u)= \\
& =\frac{1}{2} \sum_{k=0}^{N-1}\left(\bar{y}_{k}^{T} Q_{1} \bar{y}_{k}+e_{k}^{T} Q_{2} e_{k}+u_{k}^{T} R u_{k}\right)+ \\
& +\frac{1}{2}\left(\bar{y}_{N}^{T} Q_{1} \bar{y}_{N}+e_{N}^{T} Q_{2} e_{N}\right)
\end{aligned}
$$

In (3) $\bar{y}_{k}=\bar{C} x_{k}$ which is the orthogonal projection of any $x_{k}$ to $\operatorname{Ker}\left(C_{r}\right)$ if one defines $\bar{C}$ as in (4).

$$
\bar{C}_{k}=I-C_{r}^{T}\left(C_{r} C_{r}^{T}\right)^{-1} C_{r}
$$

This means that, $\bar{y}_{k}$ contains the effects of the state space region not considered in $y_{k}$ and so, this can be weighted separately with $Q_{1}$. The consideration of this extra weighting can improve Discrete Algebraic Riccati Equation (DARE) solvability properties.

Remark 1: The reason of introducing $\bar{y}_{k}$ is to inject some complementary information to the solution. The idea behind can be given as a certain amount of additional information (extra weight) under the form of a weighted generalized moment term.

Now defining the reference state as $\tilde{x}_{k}=$ $C_{r}^{T}\left(C_{r} C_{r}^{T}\right)^{-1} r_{k}$ (see [2]) and doing some algebraic manipulations, the final functional leads back to a state tracking problem as:

$$
\begin{aligned}
& J(x, \tilde{x}, u)= \\
& =\frac{1}{2} \sum_{k=0}^{N-1}\left(\left(x_{k}-\tilde{x}_{k}\right)^{T} Q\left(x_{k}-\tilde{x}_{k}\right)+u_{k}^{T} R u_{k}\right)+ \\
& +\frac{1}{2}\left(x_{N}-\tilde{x}_{N}\right)^{T} Q\left(x_{N}-\tilde{x}_{N}\right)
\end{aligned}
$$

where $Q=\bar{C}^{T} Q_{1} \bar{C}+C_{r}^{T} Q_{2} C_{r}$ and for DARE solvability, the pair $\left(Q^{1 / 2}, A\right)$ is required not to have unobservable modes on the unit circle. The tracking problem can be solved using Lagrange multiplier method, considering the dynamic equations in (1) and the initial condition $x_{0}=a$ (see [3], [4]). In the following, the further details will be omitted and only the main results are presented in the sequel.

The structure of the costate variable (Lagrange multiplier) is given by:

$$
\lambda_{k}=P_{k} x_{k}+S_{k} \tilde{x}_{k+1}-Q \tilde{x}_{k}
$$

Here $Q$ is the weighting matrix defined after (5), $P_{k}$ and $S_{k}$ has to be calculated during controller design. For simplicity define $S R_{k}=Q \tilde{x}_{k}-S_{k} \tilde{x}_{k+1}$ and so $\lambda_{k}=P_{k} x_{k}-S R_{k}$.

The boundary conditions can be given at the end of time horizon:

$$
\lambda_{N}=Q x_{N}-Q \tilde{x}_{N} \Rightarrow P_{N}=Q, S_{N}=0
$$

The final recursive calculation rules and the control input results as shown in (7) (substituting $\tilde{x}_{k}=$ $\left.C_{r}^{T}\left(C_{r} C_{r}^{T}\right)^{-1} r_{k}\right)$.

The results show that the finite time tracking control consists of the well known state feedback control $\left(P_{k}, K_{x_{k}}\right)$ and another part considering the reference signal $\left(S R_{k}\right.$ or $K_{Q_{k}}$ and $K_{S_{k}}$ ). This latter part is very similar to the forcing function applied in [1] for CT cases. Usually, $x_{k+1}$ is calculated from $u_{k}$, therefore $r_{k+1}$ is needed to be known at the $k^{t h}$ time instant. However, the term $r_{k+2}$ means the application of one step preview. Of course, all the parameters $\left(P_{k}\right.$ and $S R_{k}$ ) can be calculated only backward (see (7)). So, the reference signal has to be known over the entire time horizon. In this case, the single step preview is not a problem. In the following section the DT infinite horizon output tracking will be derived using these results. 


$$
\begin{aligned}
P_{N}= & Q \quad S R_{N}=Q C_{r}^{T}\left(C_{r} C_{r}^{T}\right)^{-1} r_{N} \\
P_{k}= & Q+A^{T} P_{k+1} A- \\
& -A^{T} P_{k+1} B\left[B^{T} P_{k+1} B+R\right]^{-1} B^{T} P_{k+1} A \\
S R_{k}= & Q C_{r}^{T}\left(C_{r} C_{r}^{T}\right)^{-1} r_{k}+ \\
& +A^{T}\left[I+P_{k+1} B R^{-1} B^{T}\right]^{-1} S R_{k+1} \\
u_{k}= & -K_{x_{k}} x_{k}+ \\
& +\underbrace{R^{-1} B^{T}\left[I+P_{k+1} B R^{-1} B^{T}\right]^{-1}}_{R B} S R_{k+1} \\
u_{k}= & -K_{x_{k}} x_{k}+K_{Q_{k}} r_{k+1}-K_{S_{k}} r_{k+2}
\end{aligned}
$$

where :

$$
\begin{aligned}
K_{x_{k}} & =R^{-1} B^{T} P_{k+1}\left[I+B R^{-1} B^{T} P_{k+1}\right]^{-1} A \\
K_{Q_{k}} & =R B \cdot Q C_{r}^{T}\left(C_{r} C_{r}^{T}\right)^{-1} \\
K_{S_{k}} & =R B \cdot S_{k+1} C_{r}^{T}\left(C_{r} C_{r}^{T}\right)^{-1}
\end{aligned}
$$

\section{THE DT, INFINITE HORIZON, LQ OPTIMAL OUTPUT TRACKING SOLUTION}

This control solution can be derived from the finite horizon results considering the $k \rightarrow \infty$ limiting case, and forcing the system to has zero steady state tracking error for constant reference signal. The infinite horizon is a special case, because usually $u_{\infty}$ will not be zero. Thus, the functionals (5) and (3) become infinitely large. However, for constant reference signals the centralized cost functional (8) with the resulting control law (15) will be finite and so, the solution optimal. Here $\bar{y}_{\infty}$ and $u_{\infty}$ are the steady state values of $\bar{y}_{k}$ and $u_{k}$ respectively.

$$
\begin{aligned}
& J_{c}\left(\bar{y}, e, u, \bar{y}_{\infty}, u_{\infty}\right)=\frac{1}{2} \sum_{k=0}^{\infty}\left(\bar{y}_{k}^{T} Q_{1} \bar{y}_{k}+e_{k}^{T} Q_{2} e_{k}+\right. \\
& \left.+u_{k}^{T} R u_{k}-\bar{y}_{\infty}^{T} Q_{1} \bar{y}_{\infty}-u_{\infty}^{T} R u_{\infty}\right)
\end{aligned}
$$

The following results will be only partially derived from the functional. Instead of the direct solution of the infinite horizon functional, an additional condition (zero steady state tracking error) will be considered. This leads to an LQ optimal state feedback stabilization part (the well known infinite horizon LQ optimal regulator) and an auxiliary term which is a feedforward part guaranteing zero steady state tracking error for constant output reference signal. The following results was derived directly from the finite horizon case taking $k \rightarrow \infty$ :

$$
\begin{aligned}
& P=Q+A^{T} P A-A^{T} P B\left[B^{T} P B+R\right]^{-1} B^{T} P A \\
& K_{x}=R^{-1} B^{T} P\left[I+B R^{-1} B^{T} P\right]^{-1} A \\
& K_{Q}=R^{-1} B^{T}\left[I+P B R^{-1} B^{T}\right]^{-1} Q C^{T}\left(C C^{T}\right)^{-1}
\end{aligned}
$$

Now, only $K_{S}$ is further needed to completely determine the control input. It was derived using the additional condition of zero steady state tracking error. At first, consider the system state dynamics using (1), (7) and (9):

$$
x_{k+1}=A x_{k}-B K_{x} x_{k}+B K_{Q} r_{k+1}-B K_{S} r_{k+2}
$$

Assume that $r_{k}=r_{\infty}=$ const $\forall k \geq 0$. Cases when only $r_{k+i}=r_{\infty}=$ const $\forall i \geq 1$ (reference signal becoming constant only after a given finite horizon) are also considered, because this needs only the change of initial state from $x(0)$ to $x(k)$ (both finite). In this way the $x_{\infty}$ steady state can be expressed using (10):

$$
\begin{aligned}
& x_{\infty}=\left(A-B K_{x}\right) x_{\infty}+B K_{Q} r_{\infty}-B K_{S} r_{\infty} \Rightarrow \\
& x_{\infty}=\left[I-A+B K_{x}\right]^{-1}\left(B K_{Q}-B K_{S}\right) r_{\infty}
\end{aligned}
$$

For the calculation of (11) the existence of the inverse matrix is needed. This needs $I-A+B K_{x}$ to has nonzero determinant. However, this determinant is the characteristic polynomial of the closed-loop system (with LQ optimal $K_{x}$ state feedback gain) at $z=1$ :

$$
\begin{aligned}
\varphi_{c}(z) & =\left.\operatorname{det}\left(z I-A+B K_{x}\right)\right|_{z=1}= \\
& =\operatorname{det}\left(I-A+B K_{x}\right) \neq 0
\end{aligned}
$$

(12) has to be satisfied in all design, because LQ optimal control places the poles of the closed-loop system inside the unit circle. So, the closed-loop will not have any $z=1$ pole and (11) can be calculated.

Now consider the steady state output and reference signals (using (2) and (11)):

$$
\begin{aligned}
r_{\infty} & =y_{\infty}=C_{r} x_{\infty}= \\
& =C_{r}\left[I-A+B K_{x}\right]^{-1} B\left(K_{Q}-K_{S}\right) r_{\infty}
\end{aligned}
$$

From (13) the condition for $K_{S}$ will be the following:

$$
\begin{aligned}
& C_{r}\left[I-A+B K_{x}\right]^{-1} B K_{S}= \\
= & C_{r}\left[I-A+B K_{x}\right]^{-1} B K_{Q}-I \\
& F K_{S}=F K_{Q}-I \\
& \text { where } F=C_{r}\left[I-A+B K_{x}\right]^{-1} B
\end{aligned}
$$

Here $F$ will be an $r \times m$ matrix because $C_{r}$ is $r \times n$ and $B$ is $n \times m$ (see the output, input and state dimensions after (1) and (2)). The calculation of $K_{S}$ depends on the dimension and rank of $F$. It can be proved that $\operatorname{rank}(F)=\min (r, m)$, so three cases have to be considered:

1) $r=m$ the number of outputs equals the number of inputs. $F$ is a full rank, quadratic, invertible matrix $K_{S}$ can be exactly calculated:

$K_{S}=K_{Q}-F^{-1}$

2) $r>m$ the number of outputs is larger than the number of inputs. $F$ is a rank $m$ rectangular matrix. Only least squares optimal solution can be obtained for $K_{S}$, which is obvious, because it is well known that, if the number of outputs is larger than the number of inputs one can not control all of them: $K_{S}=K_{Q}-\left(F^{T} F\right)^{-1} F^{T}$ 
So, in this case the stated tracking problem can not be solved.

3) $r<m$ the number of outputs is smaller than the number of inputs. $F$ is a rank $r$ rectangular matrix. An exact solution can be obtained using the MoorePenrose pseudoinverse (pinv) of $F$ :

$K_{S}=K_{Q}-\operatorname{pinv}(F)$

In this way, the infinite horizon control input can be constructed using the calculated $K_{x}, K_{Q}$ and $K_{S}$ gains:

$$
u_{k}=-K_{x} x_{k}+K_{Q} r_{k+1}-K_{S} r_{k+2}
$$

Now examine the structure of the derived control law considering the state dynamic equation (10) $(M$ depends on the size of $F$, it can be $F^{-1},\left(F^{T} F\right)^{-1} F^{T}$ or $\left.\operatorname{pinv}(F)\right)$ :

$$
\begin{array}{r}
x_{k+1}=A x_{k}-B K_{x} x_{k}+B K_{Q} r_{k+1}- \\
-B\left(K_{Q}-M\right) r_{k+2} \\
\underbrace{x_{k+1}=\left(A-B K_{x}\right) x_{k}}_{\text {Simple state feedback }}+\underbrace{B M r_{k+2}}_{P \text { term }}- \\
-\underbrace{B K_{Q}\left(r_{k+2}-r_{k+1}\right)}_{D \text { term }}
\end{array}
$$

(16) shows that, the simple state feedback is completed with a $P$ and a $D$ term for the reference signal.

However, the application of control input (15) needs to know not only $r_{k+1}$ but $r_{k+2}$ also, so needs one step preview. This can cause problems in case of real time generated reference inputs. This problem can be solved applying linear extrapolation:

$$
\begin{aligned}
& r_{k+2}=-r_{k}+2 r_{k+1} \\
& u_{k}=-K_{x} x_{k}+K_{S} r_{k}+\left(K_{Q}-2 K_{S}\right) r_{k+1}
\end{aligned}
$$

For smooth reference signals (usually real reference signals are smooth) this provides the same good tracking performance (as the one step preview) without significant increase in input energy. For step-like reference signals the tracking is also good, but significant increase occurs in input energy.

In the next section asymptotic stability, asymptotically zero tracking error and the satisfaction of the separation principle will be proven. In the following $\phi=\left[A-B K_{x}\right]$ will be used to simplify the expressions.

\section{STATEMENT AND PROOF OF THE PROPERTIES OF THE DERIVED INFINITE HORIZON CONTROL METHOD}

Theorem 1 (Guaranteed asymptotic stability): The proposed infinite horizon output tracking solutions (15) and (17) guarantee asymptotic stability for finite, constant, output reference signals.

Proof: The proof can be done in a constructive way. The simple state feedback obtained as LQ optimal has to be stable, so only the effect of the reference signal is questionable. Start with time instant 0 and assume that $r_{k}=r_{\infty}=$ const $\forall k \geq 1$. In this way, one gets from (16):

$$
x_{1}=\phi x_{0}+B M r_{\infty}
$$

both for (15) or (17) Considering (18) and the state equation, the further states result as follows:

$$
\begin{aligned}
x_{2} & =\phi^{2} x_{0}+\phi B M r_{\infty}+B M r_{\infty} \\
x_{3} & =\phi^{3} x_{0}+\phi^{2} B M r_{\infty}+\phi B M r_{\infty}+B M r_{\infty} \\
\vdots & \\
x_{n} & =\phi^{n} x_{0}+\left[\sum_{m=0}^{n-1} \phi^{m}\right] B M r_{\infty}
\end{aligned}
$$

To decide about asymptotic stability one has to examine $n \rightarrow \infty$. $\phi$ is the closed-loop system matrix which has all of its eigenvalues inside the unit circle. This means that it is a nilpotent matrix and its power achieves zero if $n \rightarrow \infty$. So, one gets:

$$
x_{\infty}=\lim _{n \rightarrow \infty}\left[\sum_{m=0}^{n-1} \phi^{m}\right] B M r_{\infty}
$$

In (20) the limes of a matrix geometric series has to be calculated. Such series converges if its matrix has all of its eigenvalues inside the unit circle. Now this is the case, so considering the limit of the convergent series the steady state results as:

$$
x_{\infty}=(I-\phi)^{-1} B M r_{\infty}
$$

The $(I-\phi)^{-1}, B$ and $M$ matrices are finite, so, for finite $r_{\infty} \quad x_{\infty}$ is finite. This means that, the system is asymptotically stable for finite, constant reference signals.

Theorem 2 (Asymptotically zero tracking error): The proposed infinite horizon output tracking solutions (15) and (17) guarantee asymptotically zero tracking error for finite, constant, output reference signals if the number of outputs is lower or equal than the number of inputs

Proof: Consider (21) and (2):

$$
\begin{aligned}
e_{\infty}= & C_{r} x_{\infty}-r_{\infty}= \\
& C_{r}(I-\phi)^{-1} B M r_{\infty}-r_{\infty}=F M r_{\infty}-r_{\infty}
\end{aligned}
$$

The value of $F M$ for the stated case is $I$ so, zero tracking error results.

In the other case $\left(\operatorname{dim}\left(y_{k}^{r}\right)>\operatorname{dim}\left(u_{k}\right)\right)$ usually $F\left(F^{T} F\right)^{-1} F^{T} \neq I$ because only a least squares optimal solution was possible. The steady state tracking error in this case will be:

$$
e_{\infty}=\left\{F\left(F^{T} F\right)^{-1} F^{T}-I\right\} r_{\infty}
$$


Finally, one has to examine the effect of state estimation, because usually all the states of the system can not be measured. This leads to the following theorem:

Theorem 3 (Satisfaction of the separation principle):

The proposed infinite horizon output tracking solutions (15) and (17) guarantee the satisfaction of separation principle for both constant and time varying reference signals and for any type of state estimator

\section{Proof:}

The equations of the DT actual state estimator are the following (it can be either deterministic or stochastic estimator):

$$
\begin{aligned}
\hat{x}_{k} & =\bar{x}_{k}+L_{o}\left(y_{k}-C \bar{x}_{k}\right) \\
\bar{x}_{k} & =A \hat{x}_{k-1}+B u_{k-1} \\
x_{k+1}^{e} & =\hat{x}_{k+1}-x_{k+1}=\left(I-L_{o} C\right) A x_{k}^{e}
\end{aligned}
$$

Here $\hat{x}_{k}$ is the estimated state and $y_{k}$ is the measured output (see (1)). The state dynamic equations of the augmented system can be constructed considering (10) and (24):

$$
\begin{aligned}
{\left[\begin{array}{l}
x_{k+1} \\
x_{k+1}^{e}
\end{array}\right]=} & \overbrace{\left[\begin{array}{cc}
A-B K_{x} & -B K_{x} \\
0 & \left(I-L_{o} C\right) A
\end{array}\right]}^{A_{a}}\left[\begin{array}{l}
x_{k} \\
x_{k}^{e}
\end{array}\right]+ \\
& {\left[\begin{array}{c}
B K_{Q} r_{k+1}-B K_{S} r_{k+2} \\
0
\end{array}\right] }
\end{aligned}
$$

From $A_{a}$ the poles of the augmented system can be calculated as $\operatorname{det}\left(z I-A_{a}\right)=$ $\operatorname{det}\left(z I-A+B K_{x}\right) \operatorname{det}\left(z I-A+A L_{o} C\right)=0$ and neither the reference signal, nor the system states affect the dynamic of the estimation error. So, the separation principle is satisfied.

Summarizing the results, the derived controller is asymptotically stable, can guarantee zero steady state tracking error and can be independently completed with state estimation.

In the following section an application example considering the trajectory tracking control of a quadrotor helicopter will be introduced, examining both the one step preview and the extrapolation cases.

\section{APPLICATION EXAMPLES WITH ONE STEP PREVIEW AND EXTRAPOLATION}

The considered quadrotor model is obtained from a joint project published in [12]. In [13] a nonlinear Matlab Simulink model of the quadrotor helicopter was constructed. In [14] a CT, LTI model is derived from this, linearizing the nonlinear model in hovering.

The states, measured outputs and inputs of the resulting model are the following:

States: $\mathrm{x}=$ [vertical position in earth coord. sys. (Z), quadrotor velocity components in body coord. sys. ( $\mathrm{u} \mathrm{v} \mathrm{w}$ ), quadrotor angular velocity components in body coord. sys. (P Q R), Euler angels $\left.\left(\begin{array}{lll}\varphi & \theta & \psi\end{array}\right)\right]$

Measured outputs: $\mathrm{y}=[$ accelerations in body coord. sys. ( $\left.\begin{array}{ccc}\dot{u} & \dot{v} & \dot{w}\end{array}\right)$, measured altitude ( $\mathrm{h}=-\mathrm{z}$ assuming flat ground), quadrotor angular velocity components in body coord. sys. (P Q R), quadrotor orientation $(\psi)]$.

Inputs: $\mathrm{u}=$ [pitching command $\delta_{\text {pitch }}$, rolling command $\delta_{\text {roll }}$, yawing command $\delta_{\text {yaw }}$, ascending / descending command $\left.\delta_{\text {asc } / \text { desc }}\right]$

This CT model was controllable and observable and it was transformed into a DT, LTI model using zero order hold discretization, after defining the sample time. The sample time was defined considering the bandwidth of the openloop system.

The open-loop bandwidth was approximately $\omega_{b}=4 \frac{\mathrm{rad}}{\mathrm{sec}}$. The closed-loop bandwidth can be an order of magnitude less then this (see [4] p. 485), and the sampling frequency has to be selected between six and forty times the closedloop bandwidth (see also [4] p. 485). So, finally five times the open-loop bandwidth was selected which resulted in the following sampling frequency and time: $\omega_{s}=20 \frac{\mathrm{rad}}{\mathrm{sec}} T=$ $0.05 \mathrm{sec}$ The discretization was done considering this sampling time. The resulted DT model is also controllable and observable. The developed algorithm with one step preview and with extrapolation was applied on this model.

The weighting of the states not included in output tracking was determined using the method of inverse squares and considering the required limits of the states. The goal was to remain in the linear range around hovering state, which can possibly provide that, the designed linear controller can work also on the nonlinear helicopter.

The considered upper limits were the following:

$0.5 \mathrm{~m} / \mathrm{sec}$ for $\mathrm{u}, \mathrm{v}, \mathrm{w}$ velocity components

$2 \mathrm{deg} / \mathrm{sec}$ for $\mathrm{P}, \mathrm{Q}, \mathrm{R}$ angular velocity components

5 deg for Euler angles

100 for input commands
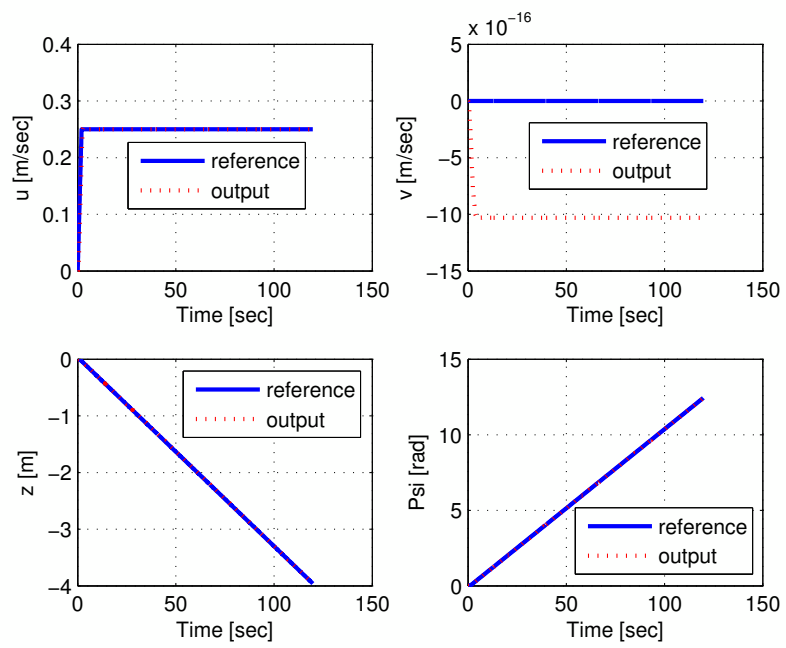

Fig. 1. Velocity, altitude and yaw angle tracking

Of course, the weighting is sometimes different, if it is needed or possible. The other weights were selected by trial and error. The measurability of all states was assumed in all cases (so the state estimator was not implemented).

The results can be qualitatively compared using the approximate two norms of control inputs and tracking errors 

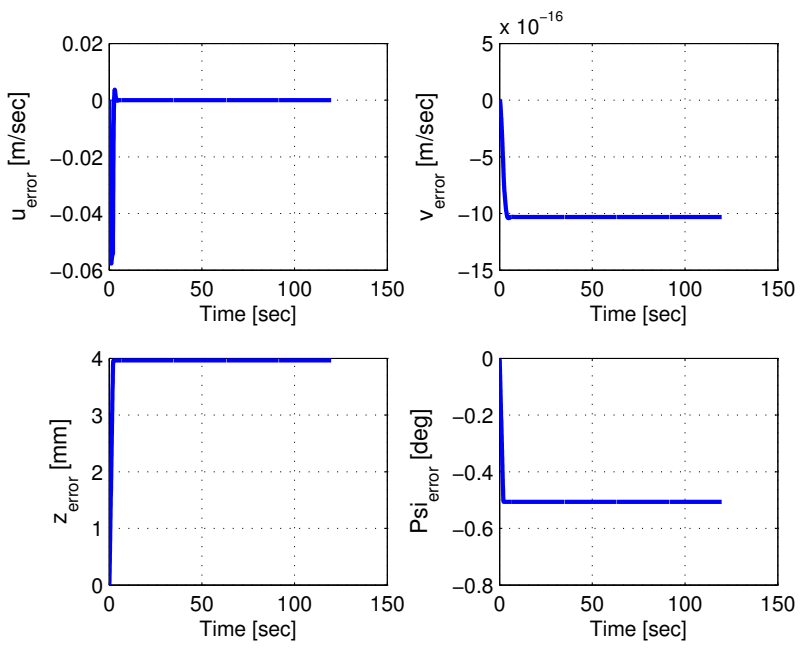

Fig. 2. Velocity, altitude and yaw angle tracking errors

TABLE I

TWO NORMS OF COMMANDS AND ERRORS

\begin{tabular}{|c||c|c|}
\hline Two norm of: & One step preview & Extrapolation \\
\hline \hline$\delta_{\text {pitch }}$ & 0.0934 & 0.1277 \\
\hline$\delta_{\text {roll }}$ & $3 \mathrm{e}-16$ & $3.93 \mathrm{e}-16$ \\
\hline$\delta_{\text {yaw }}$ & 4.1056 & 4.1465 \\
\hline$\delta_{\text {asc } / \text { desc }}$ & 0.7076 & 0.7165 \\
\hline$u_{\text {error }}$ & 0.0743 & 0.0745 \\
\hline$v_{\text {error }}$ & $1.12 \mathrm{e}-14$ & $1.12 \mathrm{e}-14$ \\
\hline$z_{\text {error }}$ & 0.0432 & 0.0432 \\
\hline$\psi_{\text {error }}$ & 0.0962 & 0.0962 \\
\hline
\end{tabular}

(the approximate two norms were calculated using trapezoid integration rule until the end of simulation (not until infinity!)).

The reference signals were the velocity components $(u, v)$ (becoming constant after a time), the vertical position $(Z)$ and the orientation $(\psi)$ (ramp-type signals),

The weighting matrices both for one step preview and extrapolation were:

$$
\begin{aligned}
& Q_{1}=<\begin{array}{llllll}
0 & 0 & 1 & 820820501351350
\end{array} \text {, } \\
& Q_{2}=<100010010010000>\text {, } \\
& \mathrm{R}=<1 \mathrm{e}-6 \text { 1e-6 1e-6 1e-6 > }
\end{aligned}
$$

The two norm results are summarized in table I. The developed control strategy works well even for the ramp-type references. This is a very promising result. The extrapolation case uses a bit more control energy (meanwhile it gives the same good tracking errors) but the difference from one step preview is not significant. So, the one step preview and extrapolation cases are almost exactly the same, which means that the use of extrapolation is a suitable solution (for smooth signals applied here). The tracking results with extrapolation are shown in Fig. 1. The tracking errors are all acceptable (see Fig. 2) so, the tracking performance is very good.

\section{CONCLUSIONS}

The paper suggests an optimal output tracking control method for DT LTI systems and for constant reference sig- nals such that the controller assures asymptotical stability and zero tracking error. It also satisfies the separation principle.

At first, the finite horizon solution was derived used as a basis for deriving the infinite horizon solution. The resulted new control method guarantees asymptotic stability and asymptotically zero tracking error, for constant reference outputs. The number of the outputs must be inferior or equal to the dimension of the input. In case of a state estimator, the separation principle is satisfied. The dynamics of the state estimator is completely independent from the controlled system states and from the reference signal (but of course, it depends from the measured outputs of the system). Originally, the method uses a one step preview of the reference signal, but for smooth signals an extrapolation can be used instead, allowing the realtime application of the method. A quadrotor trajectory tracking task has been solved in order to emphasize the importance of the above solution. The extrapolation of the reference signal has been proven to successfully replace the one step ahead prediction of the reference trajectory. What is more, not only the constant, but also the ramp-type reference signals are well tracked.

So, the need to investigate the properties for time varying references arises. Ramp-type, bounded, $l_{1}$ and $l_{2}$ signals should all have to be considered.

Further investigation can be done upon the robustness of the given method such as robustness under model uncertainty and disturbance. Another question is the performance degradation of the closed loop with jointly applied state estimator.

\section{REFERENCES}

[1] M. Athans and P. L. Falb, Optimal Control, An introduction to the Theory and its Applications, McGraw-Hill Book Company; 1966.

[2] B. D. O. Anderson and J. B. Moore, Optimal Control, Linear Quadratic Methods, Prentice Hall, Englewood Cliffs, NJ; 1989.

[3] T. Chen and B. Francis, Optimal Sampled-Data Control Systems, Springer-Verlag London Limited; 1995.

[4] G. F. Franklin, J. D. Powel and M. L. Workman, Digital Control of Dynamic Systems, Addison-Wesley Publishing Company; 1990.

[5] J. B. Burl, Linear Optimal Control, $\mathcal{H}_{2}$ and $\mathcal{H}_{\infty}$ Methods, Addison Wesley Longman Inc., Menlo Park California, 1999.

[6] M. J. Grimble and A. W. Ordys, Predictive Control for Industrial Applications, Ann. Rev. in Cont., vol. 25, 2001, pp 13-24.

[7] E. Mosca, and A. Casavola, Deterministic LQ Preview Tracking Design, IEEE Trans. on Aut. Cont., vol. 40, 1995, pp 1278-1281.

[8] E. Mosca, Optimal, Predictive and Adaptive Control, Prentice Hall; 1995.

[9] A. Farooq and D. J. N. Limebeer, "Path Following of Optimal Trajectories Using Preview Control", in Proc. of 44th IEEE Conference on Decision and Control, and the European Control Conference 2005, Seville, Spain, 2005, pp. 2787-2792.

[10] J. L. Willems and I. M. Y. Mareels, A Rigorous Solution of the Infinite Time Interval LQ Problem with Constant State Tracking, Sys. \& Cont. Let., vol. 52, 2004, pp 289-296.

[11] E. Barbieri and R. Alba-Flores, On the Infinite-horizon LQ Tracker, Sys. \& Cont. Let., vol. 40, 2000, pp 77-82.

[12] A. Soumelidis, P. Gaspar, P. Bauer, B. Lantos and Z. Prohaszka, "Design of an embedded microcomputer based mini quadrotor UAV", in Proc. of European Control Conference 2007, Kos, Greece, 2007

[13] Gy. Ritzinger, Szitakoto negyrotoros helikopter szabalyozasa allapotvisszacsatolas es allapotbecslo alkalmazasaval, MSc Thesis work in hungarian, Budapest; 2007.

[14] P. Bauer, Gy. Ritzinger, A. Soumelidis and J. Bokor, LQ Servo Control Design with Kalman Filter for a Quadrotor UAV, accepted for publication in Periodica Polytechnica Transportation Engineering in November 2007 